\title{
Investigation of the process of controlled starting of the open-pit locomotive for ensuring the maximum adhesion coefficient at the starting
}

\author{
Ambartsum Keropyan ${ }^{1, *}$, Yuriy Babichev ${ }^{1}$, and Pavel Sizin ${ }^{1}$ \\ ${ }^{1}$ NITU "MISiS", Moscow, Russia
}

\begin{abstract}
In order to control the calculated coefficient of adhesion of the locomotive bandages with the rail, a controlled starting of the career locomotive is suggested, in which the relative traction coefficient varies over the time according to the exponential dependence. To realize a rational starting process the tractive effort during the $1 / 4$ of the starting period should be $3 / 4$ of the maximum which is recommended by the traction conditions for a given section of the track, and for the remaining time, i.e. in the interval of the subsequent three-fourths of the starting time, the tractive effort must be smoothly brought to the required level, acceleration during the same period must also be changed (decreased) and at the end of the starting process, when the train reaches the working speed at this track section, it must be made equal to zero. It is proposed a relationship for determining of the starting time of the locomotive, depending on the initial parameters and actual operating conditions, which makes it possible to develop technical requirements for the automatic launch system of a open pit locomotive, which ensure the implementation of the maximum possible values of adhesion coefficients closed to optimal after the starting process.
\end{abstract}

\section{Introduction}

For the locomotives which operate in the open-pit conditions with high track gradients and with the presence of movable rails, the choice of starting mode can be crucial for realizing the maximum coefficient of adhesion in the heaviest operating mode of the locomotive when train starts from the place, especially if this occurs when leaving the trench on a ruling grade. This circumstance is especially actual with the current trend of increasing depth of quarrying [1].

Professor I.P. Isaev $[2,3]$ considered the effect of three different regimes of increasing the tractive effort (the running resistance forces were considered as quasi-constant during the starting period and were significantly lower than tractive effort) on the value of the realized adhesion coefficient.

\footnotetext{
${ }^{*}$ Corresponding author: am kerop@mail.ru
} 
The first starting mode is called the accelerated with monotonous (without inflection points) decreasing acceleration increment. The second mode is conditionally called the uniform, while the traction effort, minus the resisting forces, increases in proportion to the starting time and the third mode - accelerated with the monotonically increasing acceleration increment.

The researches $[2,4,5,6,7]$ have found that the highest adhesion coefficients during the starting are provided in the regime when the increasing of the traction effort reaches $75 \%$ of the maximum value, approximately in the first quarter of the starting time segment.

\section{Object methods of the research}

In view of the above, let's present the scheme of the starting schedule in the following form (Figure 1). Let's introduce the concept of the relative traction coefficient $\varphi=\frac{F}{F_{\max }}$ as a function of the relative starting time $\tau=\frac{t}{t_{\mathrm{n}}}$. Here, $F$ is the current value of the traction effort of the locomotive during the starting, $F_{\max }$ is the limiting value of the tractive force of the locomotive according to the adhesion conditions (at the end of the start), $t$ is the current time value during the starting, $t_{n}$ is the total start time.

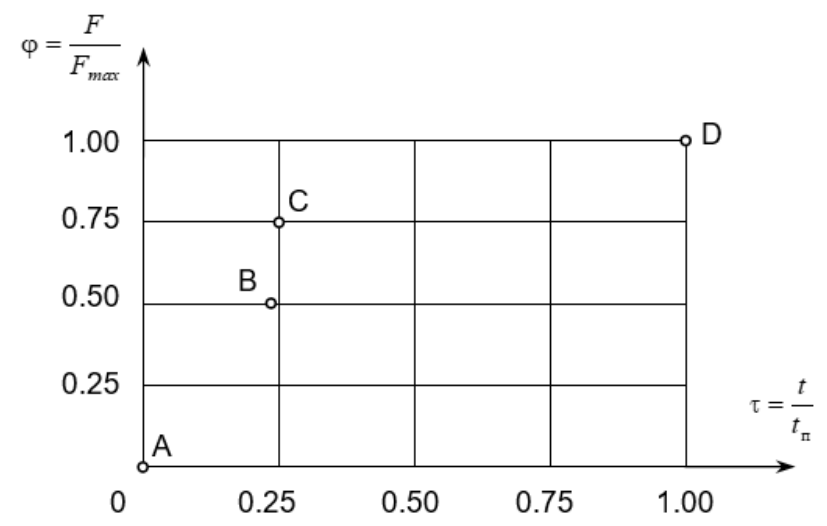

Fig. 1. The ccharacteristic points (A, B, C, D) of the dependence of the traction effort from the starting time.

The coordinates of characteristic points:

- A $(0.00 ; 0.00)$ is the origin of coordinates (according to I.P. Isaev with reference to [4]);

- B $(0.23,0.50)$ - the average point along the ordinate axis at $\mathrm{F} / \mathrm{Fmax}=0.5$ (our proposal);

- $\mathrm{C}(0.25 ; 0.75)$ - the first quarter of the starting time at $\mathrm{F}=0.75$ Fmax $[2,4]$;

- D $(1.00,1.00)$ - completion of the starting (according to Isaev [2]).

Traction effort is proportional to the acceleration, if the moving bodies do not vary in mass. Since the loaded train has a practically unchanged mass, Fig. 1 in relative units also characterizes the change in the relative acceleration $\left(a / a_{\max }\right)$ in the function of the relative time $t / t_{\Pi}$.

To determine the mathematical dependence of the relative traction coefficient on the start time, polynomial and exponential approximations were considered, at that the assumed dependence $\varphi(\tau)=\frac{F(\tau)}{F_{\max }}$ was divided into two intervals: $0<\square<0.23$ and $0.23<\square<1.00$.

The approximation of the dependence of the relative traction coefficient (i.e. the tractive effort, or the acceleration, which is the same) on the relative time during acceleration must 
pass through the characteristic points marked in Figure 3 and must take into account the physical properties of the acceleration process. In addition, from the physical considerations, the approximating function must satisfy the conditions $0 \leq \square(\square) \leq 1$ for any values of the time.

On the segment from 0 to 0.23 (Figure 1) the curve $\square(\tau)$ can be approximated by an exponential function

$$
\varphi_{11}(\tau)=0.01 \cdot \tau \cdot e^{100 \cdot \tau^{2}}+0.25 \cdot \tau
$$

On the segment from 0.23 to 1.00 we represent the approximation in the form of an exponential function

$$
\varphi_{22}(\tau)=0.5+0.44 \cdot\left[1-e^{-32 \cdot(\tau-0.23)}\right]+0.06 \cdot \tau
$$

The figure 2 shows the graphs of the polynomial and exponential dependences of the relative traction coefficient (tractive effort) in the process of starting the locomotive from the relative time.

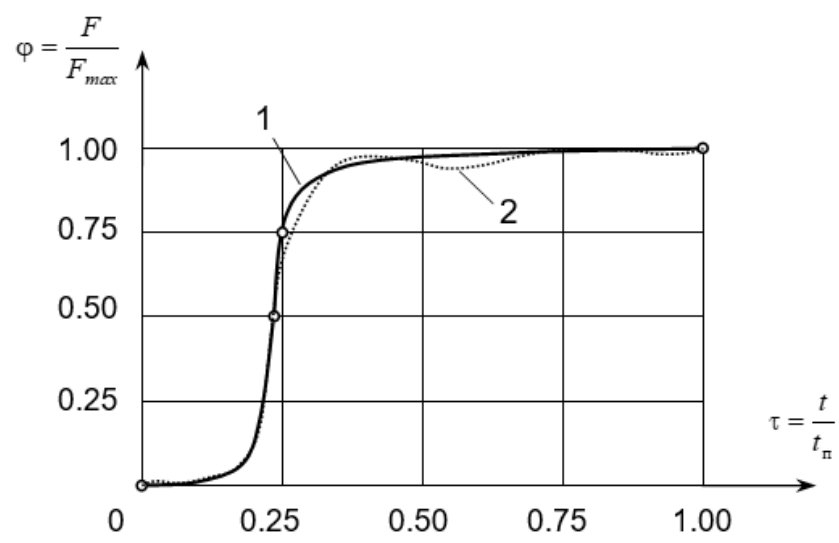

Fig. 2. The dependency graphs for exponential (1) and polynomial (2) approximations.

An analysis of the results of the theoretical studies has shown that the polynomial approximation of the powers 2, 3, 4 and 5 does not give satisfactory results, both on the whole time interval and on intermediate intervals of approximation. Therefore, for further studies the exponential approximation is taken as the basis.

As a result of integration of equation (1) in the range from 0 to $0.23 t_{\mathrm{n}}$, the approximation of the relative velocity graph was obtained:

$$
\tilde{v}_{1}(\tau)=5 \cdot 10^{-5} \cdot\left(e^{100 \cdot \tau^{2}}-1\right)+0.125 \cdot \tau^{2},
$$

By integrating (2) over the interval from $0.23 t_{\Pi}$ to $1.00 t_{\Pi}$, the approximation of the relative velocity graph was obtained:

$$
\widetilde{v}_{2}(\tau)=0,94 \cdot \tau+0,01375 \cdot e^{-32 \cdot(\tau-0.23)}+0,03 \cdot \tau^{2}-0,215
$$

In Eq. (3) and (4) $\tilde{v}_{1}(\tau)$ and $\tilde{v}_{2}(\tau)$ are the relative velocities during the starting at intervals from 0 to $0.23 t_{\text {п }}$ and $0.23 t_{\text {п }}$ to $1.00 t_{\text {п }}$ respectively.

Figure 3 shows the combined graphs of the relative acceleration and the relative speed of the controlled starting process. 


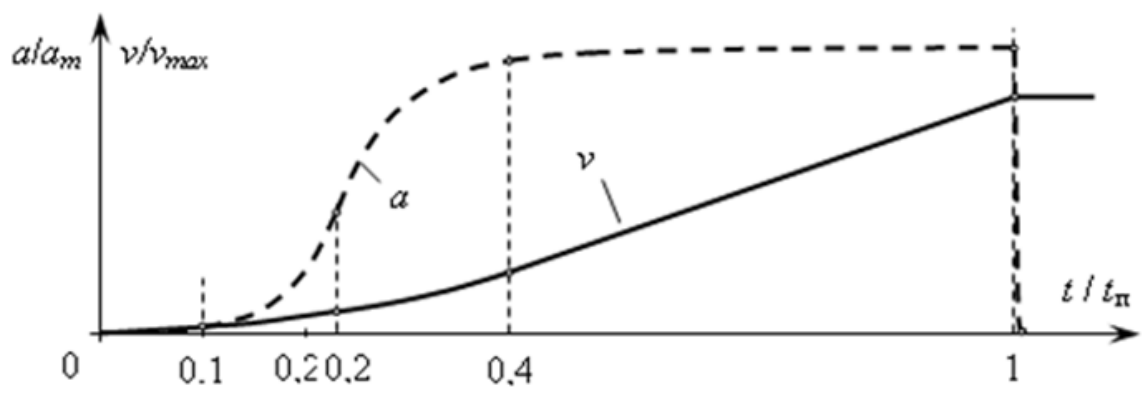

Fig. 3. Graphs of relative acceleration and relative speed of the starting process.

\section{The results of the research and their discussion}

Based on the conducted studies, it is possible to obtain a mathematical dependence of the change in the adhesion coefficient of the bandages of locomotives with the rails during the starting. For example, for a traction aggregate of the alternating current OPE 1 (for $0<\mathrm{V}<40$ $\mathrm{km} / \mathrm{h})[8,9,10,11,12]$

$$
\Psi_{\text {OPE }}=0.21+\frac{7}{53+3 \cdot V} \text {. }
$$

To determine the absolute value of the adhesion coefficient, the functions (3) and (4) are substituted in the Eq. (5) instead of the speed V.

Since the speed is calculated in relative units, we will assume that its largest value is 40 $\mathrm{km} / \mathrm{h}$, that is, $V_{\max }=\widetilde{V}(1.00)=0.755 \mathrm{~km} / \mathrm{h}$. In order to convert from relative units to absolute ones, you can add a coefficient:

$$
k_{V}=\frac{40}{0.755}=52.98, \text { then } \Psi_{O P E 1}=0,21+\frac{7}{53+3 \cdot \tilde{V}(\tau) \cdot k_{V 3}}
$$

Similar results can be obtained for direct current traction aggregate, for example PE 2M (for $0<\mathrm{V}<40 \mathrm{~km} / \mathrm{h}$ ) [5,6]

$$
\Psi_{P E}=0.225+\frac{7.2}{100+20 \cdot V}
$$

For the traction aggregates OPE 1 and PE 2M, the adhesion coefficients at the moment of starting (coefficient of friction of rest) are respectively 0.342 and 0.297 . Calculations show that when using a rational mode of controlled start after the starting, these characteristics will be respectively equal to 0.26 and 0.235 at an operating speed of $40 \mathrm{~km} / \mathrm{h}$. It should be noted that the calculations were carried out for the conditions of dry rails without the supply of sand.

Figure 4 shows the dependence of the adhesion and speed coefficients for the controlled starting of the traction aggregates OPE 1 and PE $2 \mathrm{M}$. 


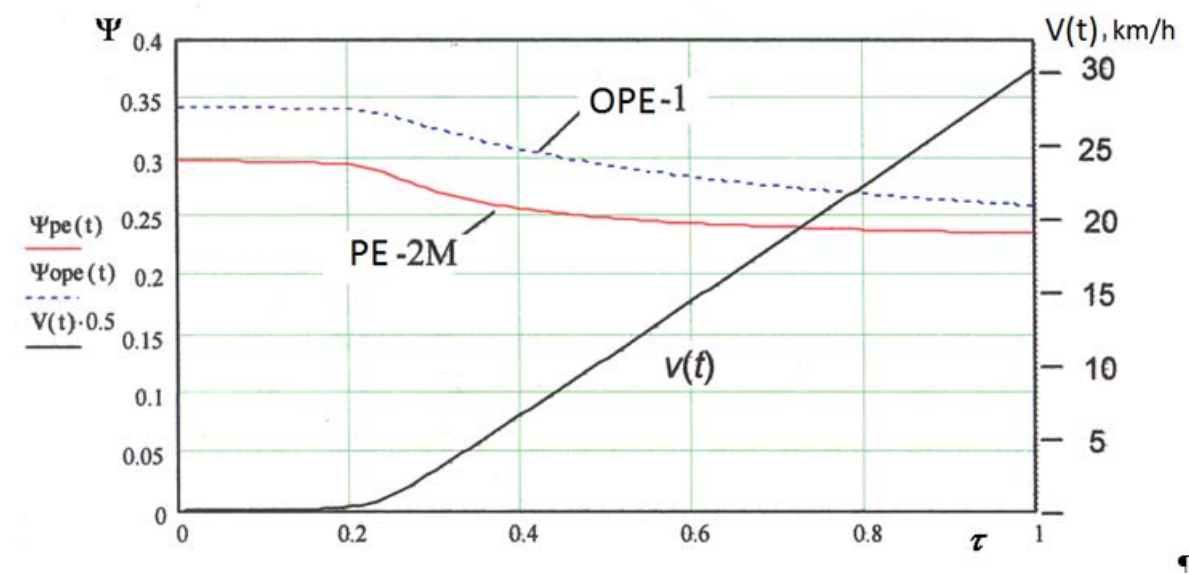

Fig. 4. Dependences of the adhesion coefficients and the speed of the traction aggregates OPE 1 and PE 2 during the starting process.

Application of the developed mathematical model of controlled starting of the open-pit locomotives allows to determine the optimum value of its starting time.

The differential equation of motion of locomotive is

$$
M_{\text {пр }} \frac{d V(t)}{d t}=M_{\text {пр }} \cdot a(t)=F_{\text {Л }}(t)-F_{\text {сопр }}(t),
$$

where $F_{\text {л }}(t)$ is the current value of tractive effort of the locomotive, N;

$F_{\text {conp }}(t)$ is the resultant of forces of resistance to movement of locomotive, N;

$M_{\text {пр }}$ is the reduced mass of the locomotive, $\mathrm{kg}$, defined as

$$
M_{\text {пр }}=\frac{(1+\gamma)}{g}\left(P_{\text {сц }}+Q\right),
$$

where $P_{\text {сц }}$ is the coupling weight of the locomotive, N;

$\mathrm{Q}$ is the weight of the trailing part of the train. N;

$\gamma$ is the inertia coefficient of the rotating masses of the locomotive, $\gamma=0.06$;

$\mathrm{g}=9.81 \mathrm{~m} / \mathrm{s} 2$ - acceleration of gravity.

Substituting the corresponding values in (7), we obtain the differential equation of motion of the locomotive

$$
\frac{(1+\gamma)}{g}\left(P_{c u}+Q\right) \cdot a(t)=F_{n}(t)-F_{\text {conp }}(t) .
$$

To simplify the entries, it is assumed that $F(t)=F_{\text {Л }}(t), F_{\text {сопр }}(t)=W=$ const, then

$$
M_{n p} \cdot a(t)=F_{n}(t)-W
$$

After integrating (10) the equation for calculation of the working speed $v_{\text {раб }}$ at the end of the descent is obtained.

$$
\nu_{\text {раб }}=\frac{t_{\text {пуск }} \cdot g}{(1+v)\left(P_{c u}+Q\right)}\left\{\int_{0}^{0.23} F(\tau) d \tau+\int_{0.23}^{1} F(\tau) d \tau-F_{\text {сопр }}\right\},
$$


Where $\tau=\frac{t}{t_{\text {пуск }}}$ is the relative dimensionless current time of starting.

Substituting the values of $v_{\mathrm{p}}$ and $F_{\max }$ in (11) when the $F_{\text {conp }}$ is known, and performing the integration, we obtain the equation for the calculation of the starting time of the open-pit locomotive

$$
t_{\text {п }}=\frac{(1+\gamma)\left(P_{c u}+Q\right) \cdot v_{\mathrm{p}}}{g \cdot\left(0.763 \cdot F_{\text {max }}-F_{\text {сопр }}\right)},
$$

where $\mathrm{F}_{\max }$ is the maximum tractive effort developed by the locomotive at the time of completion of the starting process, which is ensured by the adhesion conditions after reaching the working (calculated) speed.

At the end of the speeding-up the acceleration should become zero, and the speed should be constant, for this it is necessary to decrease the tractive effort from $F_{\max }$ to $F_{\text {conp }}=W$ and the speed will become equal to the specified operating speed of the locomotive.

\section{Conclusions}

1. In order to control the calculated adhesion coefficient of the locomotive bandages with the rail, a controlled starting of the open-pit locomotive in which the relative traction coefficient varies according to the relative time according to the exponential dependence is suggested.

2. The equation for the determining of the starting time of the locomotive, depending on the initial parameters and actual operating conditions is obtained

3. Obtained dependencies allow to develop the technical requirements for the system of automatic start of the open-pit locomotive, which ensure the implementation of the maximum possible values of the adhesion coefficients, which are close to optimal, after the starting process is completed.

4. To realize a rational starting process the tractive effort during the $1 / 4$ of the starting period should be $3 / 4$ of the maximum which is recommended by the traction conditions for a given section of the track, and for the remaining time, i.e. in the interval of the subsequent three-fourths of the starting time, the tractive effort must be smoothly brought to the required level, acceleration during the same period must also be changed (decreased) and at the end of the starting, when the train reaches the working speed at this track section, it must be made equal to zero

\section{References}

1. Fesenko S.L. Parameter justification and development of technical means of railway transport systems with track gradients of $60 \%$ in deep pits. / Diss. on the Doctor of Technical Sciences (M.:,1991)

2. Isaev I.P. Random factors and coefficient of adhesion (M .: Transport. 1970)

3. Isaev I.P., Luznov Yu.M., Problems of traction of wheels of a locomotive with rails (M.:Mashinostroenie, 1985)

4. Lucas H., Wojtas B. Automatic wheelslip control. The Institution of Locomotive Engineers, vol. 56 (part № 5), 1966/67.

5. A. Keropyan, A. Gerasimova, K. Goloshapov. Influence of the track gradient on the contact temperature at the wheel-rail zone for open-pit locomotives, MATEC Web of Conferences, 129, 06009 (2017). 
6. A. Keropyan, S. Gorbatyuk, A. Gerasimova. Tribotechnical aspects of wheel-rail system interaction. International Conference on Industrial Engineering, Procedia Engineering, 206, pp. 564-569 (2017)

7. A.M. Keropyan, L.I.Kantovich, B.V.Voronin, D.A.Kuziev, V.V.Zotov. Influence of uneven distribution of coupling mass on locomotive wheel pairs, its tractive power, straight and curved sections of industrial rail tracks. IOP Conf. Series: Earth and Environmental Science 87, 062005 (2017)

8. Evdokimov B.A., Zabelin GD et al., Rail transport of opencast mining (M.:Nedra.1984)

9. Luzhnov Yu.M., Nanotribology of clutch wheels with rails (M .: Intext, 2009)

10. Keropyan A., Gerasimova A., Connection of the temperature in contact area of the wheel-rail system with the railway slope of industrial railway transport, Izvestia. Ferrous Metallurgy, National University of Science and Technology MISIS, volume 60, No5, pp. 355-363 (2017) DOI: http://dx.doi.org/10.17073/0368-0797-2017-5-355-363.

11. A.A. Gerasimova, A.M. Keropyan, A.M. Girya. Study of the Wheel-Rail System of Open-Pit Locomotives in Traction Mode, ISSN 1052-6188, Journal of Machinery Manufacture and Reliability, Vol. 47, № 1, pp. 35 - 38. (2018)

12. R. K. Basov, A. M. Keropyan. Justification of the relevance of continuous monitoring of the geometric characteristics of rolling surfaces of the career track // GIAB, No. 1, (2018). DOI: 10.25018 / 0236-1493-2018-1-0-58-66. 